\title{
L'AGRESSION SEXUELLE ENVERS LES ENFANTS. HÉBERT, M ; CYR, M. ; TOURIGNY, M. (DIR.) 2011. TOME 1. PRESSES DE L'UNIVERSITÉ DU QUÉBEC
}

\author{
Q. Bullens
}

Office de la Naissance et de l'Enfance | Carnet de notes sur les maltraitances infantiles

2013/1 - N $\mathrm{N}^{\circ} 131$

pages 21 à 21

ISSN

Article disponible en ligne à l'adresse:

http://www.cairn.info/revue-carnet-de-notes-sur-les-maltraitances-infantiles-2013-1-page-21.htm

Pour citer cet article :

Bullens Q., « L'agression sexuelle envers les enfants. Hébert, M ; Cyr, M. ; Tourigny, M. (Dir.) 2011. Tome 1. Presses de l'Université du Québec »,

Carnet de notes sur les maltraitances infantiles, $2013 / 1 \mathrm{~N}^{\circ} 131$, p. 21-21.

Distribution électronique Cairn.info pour Office de la Naissance et de l'Enfance.

(c) Office de la Naissance et de l'Enfance. Tous droits réservés pour tous pays.

La reproduction ou représentation de cet article, notamment par photocopie, n'est autorisée que dans les limites des conditions générales d'utilisation du site ou, le cas échéant, des conditions générales de la licence souscrite par votre établissement. Toute autre reproduction ou représentation, en tout ou partie, sous quelque forme et de quelque manière que ce soit, est interdite sauf accord préalable et écrit de l'éditeur, en dehors des cas prévus par la législation en vigueur en France. II est précisé que son stockage dans une base de données est également interdit. 


\section{L'AGRESSION SEXUELLE ENVERS LES ENFANTS \\ Hébert, M ; Cyr, M. ; Tourigny, M. (Dir.) 2011. Tome 1. Presses de l'Université du Québec.}

S'il est peu probable que ce premier tome dirigé par Martine Hébert, Mireille Cyr et Marc Tourigny devienne votre livre de chevet préféré, il n'en demeure pas moins un outil d'une incroyable richesse pour tout professionnel concerné dans sa pratique, de près ou de loin, par la question de l'agression sexuelle envers les enfants. Par on ne sait quelle alchimie et malgré la présence de 17 contributeurs différents, ce livre-outil déploie un style constant sans fioritures et d'une précision chirurgicale, reflet de l'engagement et du professionnalisme bien connu des différents centres impliqués dans sa rédaction.

Au travers de 11 chapitres, les auteurs nous dressent une cartographie de l'état des connaissances en la matière. La mise en perspective permettant de «placer le décor » fait immédiatement place à un chapitre consacré à la parole de l'enfant, comme pour dire qu'après avoir vu ce dont on parle, il est indispensable de se rappeler de qui et pour qui on parle. Qui est l'enfant et comment ses capacités et son développement doivent guider le travail des professionnels dans la prise en charge de sa parole (quelle qu'elle soit) et ce, qu'il s'agisse du volet judiciaire ou psychologique ? Et puisque le champ de l'agression sexuelle ne peut se limiter à ces disciplines, c'est par un point de vue médical et médicolégal que se poursuit l'ouvrage. On comprend alors comment ces différentes perspectives, clivées sur le terrain professionnel, n'interrogent pourtant qu'une seule et même réalité, celle de l'enfant (supposé) victime. Les auteurs s'intéressent ensuite aux profils et évaluations de ces derniers ainsi qu'à ceux de leurs parents, interrogeant également les effets des interventions les concernant. Les comportements sexuels problématiques chez les moins de 12 ans sont également abordés avant de mettre les projecteurs sur deux méthodes de traitement et enfin sur la prévention.

Si chacune de ces différentes parties est étayée par une bibliographie approfondie, le relevé de toutes ces études, bien que dressant un important panel de l'état des connaissances, peut parfois donner au lecteur un sentiment de confusion voire de contradiction entre les différentes déductions issues de la recherche. Loin de devoir nous bloquer dans nos réflexions, cet aspect des choses doit nous donner à penser tant le fond que la forme, les contenus des recherches et leurs méthodes. Les connaissances scientifiques et les pratiques cliniques se doivent d'être en mouvement et c'est là une des grandes qualités de cet ouvrage. Bien que nous apportant un important bagage de savoir, il nous montre dans le même temps que rien n'est figé et que le devoir du professionnel, scientifique ou clinicien, est de pouvoir continuer à avancer avec et en interro- geant le doute sur base des éléments mis en lumière. S'il nous semble qu'au-delà des études sur l'agression sexuelle, le livre pose la question des méthodologies scientifiques et des limites de l'approche hypopthético-déductive, sur le plan clinique, nous pensons qu'il met aussi en exergue la question des invariants que les pratiques pourraient connaître. Enfin, l'accent mis sur le travail avec le parent non-agresseur comme ressource tant pour l'enfant que pour le système familial et thérapeutique, nous transporte bien au-delà des considérations anciennes où le seul adulte susceptible d'aider l'enfant était son agresseur, par la reconnaissance ou non des faits.

Un livre important, utile, à lire et à interroger, à partager entre collègues. Il nous assied quelques instants sur ce que nous savons, nous aide à faire le point mais nous relève tout aussi vite vers les pistes à explorer, le travail à accomplir et les lectures à découvrir... comme le tome 2 par exemple.

\section{Q. Bullens}

Psychologue à l'équipe SOS Enfants du SAILFE (Ciney) et chercheur à l'Université de Liège.

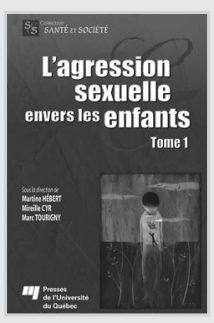

Martine Hébert, Ph.D. en psychologie, est professeure au Département de sexologie de l'Université du Québec à Montréal.

Mireille Cyr, Ph.D. en psychologie, est professeure au Département de psychologie de l'Université de Montréal.

Marc Tourigny, Ph.D. en psychologie, est professeur au Département de psychoéducation de l'Université de Sherbrooke. 